\title{
Review
}

\section{Hyaluronidase in Neuroplasty: A Review}

Standiford Helm, II, MD'1, and Gabor B. Racz, MD²

From: ${ }^{1}$ Medical Director, The Helm Center for Pain Management, Laguna Woods,

CA;

drhelm@thehelmcenter.com ${ }^{2}$ Professor and Chairman Emeritus, Texas Tech. University Health Sciences Center, Lubbock, TX; Mail4gbr@epimed.com

Address Correspondence: Standiford Helm II, MD 24902 Moulton Parkway, Suite

Laguna Woods, CA 92637 E-mail: drhelm@thehelmcenter.com

Disclaimer: Dr. Helm reports stock ownership in StimWave. Dr. Racz reports stock ownership in Halozyme and StimWave. He receives honoraria from Epimed and family members have an equity interest in Epimed. Conflict of interest: Each author certifies that he or she, or a member of his or her immediate

family, has no commercial association (i.e., consultancies, stock ownership, equity interest, patent/licensing arrangements, etc.) that might pose a conflict of interest in connection with the submitted manuscript.

Manuscript received: 03-27-2019 Revised manuscript received: 05-05-2019

Accepted for publication: 06-04-2019

Free full manuscript: www.painphysicianjournal.com
Background: Neuroplasty, also known as percutaneous adhesiolysis, is an effective treatment for persistent axial and radicular pain.

Objectives: One issue of concern is whether hyaluronidase should be used when performing neuroplasty. The objective of this narrative review is to evaluate the current literature relating to hyaluronidase and its role in neuroplasty.

Methods: The literature relating to hyaluronidase was examined via a search of PubMed and Google Scholar until April 2019, review of the citations of relevant literature, and the authors' knowledge of the literature and activity in the field. The literature was reviewed in light of hyaluronidase's physiologic role, allergenicity, medical uses, and evaluation specifically for neuroplasty.

Results: Hyaluronidase facilitates the spread of medications in the extracellular matrix by breaking down polysaccharides in the interstitial space. While allergic reactions to hyaluronidase have been reported, these reactions occurred with animal-derived preparations. The current human recombinant hyaluronidase does not have any reports of allergic reactions. Laboratory studies show that it does not evoke an immune response. Hyaluronidase has been extensively used in a variety of medical applications, including intrathecal treatment of arachnoiditis.

There have been multiple studies reporting benefit from the use of hyaluronidase in interventional procedures. One randomized trial specifically looking at the use of hyaluronidase in neuroplasty found that the addition of hyaluronidase improved pain ratings at 12 months compared to other techniques and decreased the number of procedures needed to gain effect.

Limitations: There have been limited studies examining the enhanced efficacy of neuroplasty when hyaluronidase is added.

Conclusions: Because of enhanced efficacy and safety, and because of the decrease in the number of procedures needed to be performed, hyaluronidase should be considered when deciding which medications to use when performing neuroplasty.

Key words: Neuroplasty, adhesiolysis, hyaluronidase, spinal stenosis, failed back surgery synderome, post lumbar surgery syndrome

Pain Physician 2019: 22:E555-560 europlasty, previously known as percutaneous adhesiolysis, is an effective procedure for the treatment of refractory axial and radicular pain, failed back syndrome, and spinal stenosis (1). A systematic review found Level 1 evidence supporting neuroplasty for back and lower extremity pain (2). Subsequent to the systematic review, additional studies from Korea, looking at 169 patients over a 12-month follow-up, have shown neuroplasty's efficacy in spinal stenosis $(3,4)$. A recent review of neuroplasty (5) 
reported 3 months' relief from these studies, rather than the 12 months reported in the original papers. Of the 169 patients, only 3 went on to surgery. The extent of stenosis is not a limiting factor, although foraminal runoff is (6). Regression analysis showed that the presence of a high-intensity zone on magnetic resonance imaging (MRI) was associated with favorable outcomes at 12 months (7). This finding may be associated with the role of adhesions between the posterior longitudinal ligament and dura in producing pain. Neuroplasty's efficacy is believed to be based upon both lysis of friable adhesions and ablation of inflamed innervated tissues (5).

Since neuroplasty was first developed in the 1980s, there has been discussion as to how the procedure should be performed, discussion sufficiently deeprooted that there are 2 Current Procedural Terminology (CPT) codes for the procedure: 62263 for a 2- or moreday procedure and 62264 for a one-day procedure. The CPT definition of neuroplasty is "percutaneous lysis of epidural adhesions using solution injection (e.g., hypertonic saline, enzyme) or mechanical means (e.g., catheter) including radiologic localization..." While this definition does not mandate the use of a springwound catheter, the authors believe that the use of a spring-wound catheter is inherent in the definition of neuroplasty. There are ongoing differences in understanding as to where the catheter should be placed and which medications should be injected through it. The multiplicity of protocols used in the published studies regarding neuroplasty highlights this variation.

One of the points of variation in how neuroplasty is performed is whether and how to use hyaluronidase. A 2012 systematic review indicated that hyaluronidase did not improve outcomes in neuroplasty (8). A 2014 review found some support for using hyaluronidase (9). A 2017 editorial, which questioned whether the evidence supporting the use of neuroplasty was convincing, also questioned whether hyaluronidase should be used (10). A recent systematic review performed by methodologists looking at neuroplasty for failed back surgery syndrome noted that technical characteristics, including the use of hyaluronidase, varied by the individual practitioner (11). This Brazilian review indicated that no cost-effectiveness studies of neuroplasty had been performed for this indication. A randomized controlled trial (RCT) of neuroplasty for failed back surgery syndrome found that the cost-effectiveness of a one-year quality-of-life improvement was $\$ 2,080$ (12).
The purpose of this current review is to assess the role of hyaluronidase in neuroplasty.

The review was performed via PubMed and Google Scholar searches of hyaluronidase and epiduroscopy or adhesiolysis until April 2019, review of the citations of relevant literature, and the authors' knowledge of the literature and activity in the field. The literature was reviewed considering hyaluronidase's physiologic role, allergenicity, medical uses, and evaluation specifically for neuroplasty.

Hyaluronidase has had multiple uses in medicine, including ophthalmic anesthesia, treatment of carpal tunnel syndrome, insulin absorption, subcutaneous infusion of IgG, and cancer treatment (13-17).

Hyaluronidase was first described by DuranReynals $(18,19)$ at the Rockefeller Institute. Hyaluronidase is found in tissues such as the testes and in snake and bee venoms, with functions as diverse as enhancing sperm fertilization of eggs and the spreading of toxins $(20,21)$. Hyaluronidase liquefies the interstitial barrier between cells, thus enhancing the spread of substances through the extracellular matrix. The primary mode of action is lysis of hyaluronic acid, although other components of the extracellular matrix are also substrates (22). Hyaluronic acid is a linear mucopolysaccharide consisting of alternating monosaccharides (23). Hyaluronic acid is a viscous lubricating substance with a high affinity for water. Hyaluronidase breaks hyaluronic acid into tetrasaccharides, allowing dispersion through the extracellular matrix, but not across fascial planes.

Hyaluronidase continues to be effective when combined with the medications used in interventional procedures (24).

The use of hyaluronidase as an adjunct to pain management procedures was first reported by Moore (25) in 1950. Moore found that hyaluronidase was most effective in procedures involving infiltration of subcutaneous tissues, such as hernia repairs.

There has been considerable interest in the use of hyaluronidase since Moore's pioneering work. Geurts and McCleane $(26,27)$, in uncontrolled studies, reported good relief from caudal injections and epiduroscopy using hyaluronidase.

Yousef et al (28) performed an interesting prospective, randomized, double-blinded study on failed back patients comparing $40 \mathrm{~mL}$ caudal injections, without a catheter, of local anesthetic, steroid, and $3 \%$ saline with and without hyaluronidase. Only the hyaluronidase group had significant relief at one year. Choi et al 
(29) has shown that $5 \%$ saline is as effective as $10 \% ; 3 \%$ has not been studied.

Kim et al (30) prospectively evaluated 100 patients with back or leg pain attributed to herniated lumbar discs. The patients received interlaminar epidural injections with either local anesthetic and steroid; local anesthetic and hyaluronidase; or local anesthetic, steroid, and hyaluronidase. While all groups responded well initially, only the local anesthetic, steroid, and hyaluronidase group maintained that relief through 12 weeks.

Rahimzadeh et al (31) reported the results of a prospective randomized trial of 25 patients with failed back syndrome who received transforaminal epidural steroid injections of local anesthetic and steroid with and without hyaluronidase. A significantly greater percentage of patients who received hyaluronidase had more than $50 \%$ relief at 4 weeks.

Rapcan et al (32) recently compared epiduroscopy with mechanical lysis of adhesions with and without local anesthetic and hyaluronidase in 45 patients with failed back syndrome. Only the hyaluronidase/local anesthetic group had sustained relief of low back, but not leg, pain at 12 months. In an accompanying editorial, Jamison and Cohen (33) questioned whether the results could be replicated, given the small sample size.

Gerdesmeyer et al (34) published the one year follow-up of a landmark true placebo, randomized, blinded study comparing 3-day adhesiolysis with hyaluronidase to a procedure in which the catheter was placed outside of the sacral cornu. At 12 months, there was a striking and statistically significant difference in the number of patients with greater than $50 \%$ improvement in pain and in the Oswestry Disability Index in the treatment group. This study provides Level 1 evidence of the efficacy of neuroplasty.

When Moore evaluated the use of hyaluronidase in pain management, he noted that allergic reactions may occur. There have since been 7 reported cases of allergic reaction to hyaluronidase (35-38). The reported incidence of allergies of 1:2000 seems improbably high. The types of injections at which these reactions occurred included caudal epidural, knee, and elbow injections. The authors suggest that these were IgE- and possibly T-cell-mediated allergic responses. The formulations of hyaluronidase used were animal-derived, bovine or ovine, with possible protease or immunoglobulin contamination that could provide a basis for allergic responses. These mammalian preparations have been replaced with recombinant human $\mathrm{PH} 20$ hyaluronidase. There have been no reported cases of allergic reaction since the transition to the recombinant preparation (39).

Two independent laboratories, in Brazil and China, have independently shown that hyaluronidase does not elicit an immune response. Fronza et al (40) showed that bovine hyaluronidase had anti-inflammatory potential, inhibiting cellular recruitment, edema formation, and pro-inflammatory mediator production; and suggested that hyaluronidase could be used to decrease the effects of acute inflammation. Huang et al (41) found that recombinant hyaluronidase both lacked the ability to generate inflammation and inhibited inflammation by inhibiting neutrophil infiltration. Rosengren et al (42), in a manufacturer-funded study, found that recombinant human $\mathrm{PH} 20$ hyaluronidase induced only modest immunogenicity, which had no association with adverse events.

There is currently no recommendation that skin testing be performed prior to the use of hyaluronidase.

Hyaluronidase has no known adverse effects in the human body. Birkenmaier et al (43) evaluated the effects of bupivacaine, hyaluronidase, a corticosteroid, and hypertonic saline on fibroblast proliferation. Hyaluronidase had no effect on fibroblast proliferation. Schulze, in another in vitro study, found that hyaluronidase was effective with all combinations of drugs used in neuroplasty (44).

Hyaluronidase is safe when administered intrathecally. There is extensive experience reported from Bangalore spanning years regarding the intrathecal use of hyaluronidase to treat arachnoiditis caused by both tuberculous and by noninfectious etiologies (45-47). In like manner, perineural injection of hyaluronidase did not affect the myelin or axons (48).

A recent prospective trial from Croatia of neuroplasty using hyaluronidase and normal saline for both failed back surgery syndrome and lumbar radiculopathy concluded that neuroplasty with hyaluronidase and steroid should be the first treatment choice in these patients after conservative therapy, as the procedure was simple, safe, and effective (49).

The question of the role of hyaluronidase in neuroplasty was specifically evaluated by Heavner et al (50) in 1999. Eighty-three patients with low back and leg pain and with a filling defect on epidurogram were randomized to have neuroplasty with 4 treatment regimens: hyaluronidase and hypertonic saline, hypertonic saline, isotonic saline, and hyaluronidase and isotonic saline. At 12 months, the hyaluronidase and hypertonic saline resulted in the greatest improvement in pain 
score and the Short Form McGill Pain Questionnaire. Further, the hyaluronidase/hypertonic saline group had the fewest number of procedures. Thus, the use of hyaluronidase and hypertonic saline had both the greatest benefit and the fewest interventions, compared to hypertonic saline alone or isotonic saline with or without hyaluronidase.

\section{LiMitaTIONS}

While the efficacy of hyaluronidase has been documented (2), most irrefutably by Gerdesmeyer et al's (34) randomized controlled trial, there have been limited studies examining the enhanced efficacy of neuroplasty when hyaluronidase is added.

\section{Conclusion}

Hyaluronidase acts by breaking down the viscous mucopolysaccharides in the interstitial matrix, allowing the greater spread of substances injected into the matrix. It is safe in the human body and has been widely used in medicine, including in the subarachnoid space.
Recombinant hyaluronidase has been shown to not cause an immune reaction. There is no need to perform a skin test prior to using hyaluronidase.

Two high-quality studies have shown the effectiveness of the addition of hyaluronidase to neuroplasty procedures, with one of the studies specifically looking at whether the addition of hyaluronidase improved outcomes. Several other lower-quality studies have shown benefit from adding hyaluronidase to a variety of pain management procedures.

Hyaluronidase has been shown to be safe and effective in neuroplasty. Because of enhanced efficacy and safety and because of the decrease in the number of additional procedures, hyaluronidase should be considered for all neuroplasty procedures.

\section{Acknowledgments}

We wish to acknowledge the editorial staff of Pain Physician journal for their expert assistance in improving this manuscript.

\section{References}

1. Racz GB, Heavner JE, Noe CE, et al. Epidural lysis of adhesions and percutaneous neuroplasty. In: Racz GB, Noe CE (eds). Techniques of Neurolysis. 2nd ed. Switzerland, Springer International Publishing, 2016, pp. 119-143.

2. Helm S, Racz GB, Gerdesmeyer L, et al. Percutaneous and endoscopic adhesiolysis in managing low back and lower extremity pain: A systematic review and meta-analysis. Pain Physician 2016; 19:E245-E281.

3. Moon DE, Park HJ, Kim YH. Assessment of clinical outcomes of cervical epidural neuroplasty using a Racz-catheter and predictive factors of efficacy in patients with cervical spinal pain. Pain Physician 2015; 18:E163-E170.

4. Han Y-J, Lee MN, Cho MJ, Park HJ, Moon $\mathrm{DE}, \mathrm{Kim} \mathrm{YH}$. Contrast runoff correlates with the clinical outcome of cervical epidural neuroplasty using a Racz catheter. Pain Physician 2016; 19:E1035-E1040.

5. Helm S, Knezevic N. A review of the role of epidural percutaneous neuroplasty [published online ahead of print November 30, 2018]. Pain Manag. doi: 10.2217/pmt-2018-0042.
6. Park $\mathrm{CH}$, Lee $\mathrm{S}-\mathrm{H}$, Lee S-C. Preliminary results of the clinical effectiveness of percutaneous adhesiolysis using a Racz catheter in the management of chronic pain due to cervical central stenosis. Pain Physician 2013; 16:353-358.

7. Moon SH, Lee Jl, Cho HS, Shin JW, Koh WU. Factors for predicting favorable outcome of percutaneous epidural adhesiolysis for lumbar disc herniation. Pain Res Manag 2017; 2017: 1494538.

8. Helm S, Benyamin RM, Chopra P, Deer TR, Justiz R. Percutaneous adhesiolysis in the management of chronic low back pain in post lumbar surgery syndrome and spinal stenosis: A systematic review. Pain Physician 2012; 15:E435-E462.

9. Lee K, Jamison DE, Hurley RW, Cohen SP. Epidural lysis of adhesions. Korean J Pain 2014; 27:3-15.

10. Bhatia A, Nelson A, Cohen SP. Breaking bad (tissue): Epidural adhesiolysis and its outcomes. Anesth Analg 2017; 124:1755-1777.

11. Brito-García N, García-Pérez L, Kovacs FM, et al. Efficacy, effectiveness, safety, and cost-effectiveness of epidural adhesiolysis for treating failed back surgery syndrome. A systematic review. Pain Med 2018; 20:692-706.

12. Manchikanti L, Pampat V, Bakhit CE, Pakanati RR. Non-endoscopic and endoscopic adhesiolysis in post lumbar laminectomy syndrome: A one-year outcome study and cost effectiveness analysis. Pain Physician 1999; 2:52-58.

13. Leffmann R. Hyaluronidase in carpal tunnel syndrome. Lancet 1964; 284:411.

14. Muchmore DB, Vaughn DE. Accelerating and improving the consistency of rapid-acting analog insulin absorption and action for both subcutaneous injection and continuous subcutaneous infusion using recombinant human hyaluronidase. J Diabetes Sci Technol 2012; 6:764-772.

15. Osgood RJ, Skipper JF, Cowell JA, et al. Abstract B86: Pegylated recombinant human hyaluronidase $\mathrm{PH}_{20}$ (PEG$\mathrm{PH} 2 \mathrm{O}$ ) enhances nab-paclitaxel plus gemcitabine efficacy in human pancreatic cancer xenografts. Cancer Res 2015; 75:B86-B86.

16. Wasserman RL. Overview of recombinant human hyaluronidase-facilitated subcutaneous infusion of IgG in primary immunodeficiencies. Immunotherapy 
$2014 ; 6: 553-567$.

17. Hompesch M, Muchmore DB, Morrow L, Vaughn DE. Accelerated insulin pharmacokinetics and improved postprandial glycemic control in patients with type 1 diabetes after coadministration of prandial insulins with hyaluronidase. Diabetes Care 2011; 34:666-668.

18. Duran-Reynals F. The effect of extracts of certain organs from normal and immunized animals on the infecting power of vaccine virus. J Exp Med 1929; 50:327-340.

19. Duran-Reynals F. Exaltation de l'activité du virus vaccinal par les extraits de certains organes. $C R$ Seances Soc Biol Fil 1928; 9:6-7.

20. Duran-Reynals F. A spreading factor in certain snake venoms and its relation to their mode of action. J Exp Med 1939; 69:69-81.

21. Chain E, Duthie E. Identity of hyaluronidase and spreading factor. $\mathrm{Br}$ ] Exp Pathol 1940; 21:324.

22. Watson D. Hyaluronidase. Br J Anaesth 1993; 71:422-425.

23. Necas J, Bartosikova L, Brauner P, Kolar J. Hyaluronic acid (hyaluronan): A review. Vet Med (Praha) 2008; 53:397-411.

24. Schulze C, Bittorf T, Walzel H, Kundt G, Bader R, Mittelmeier W. Experimental evaluation of hyaluronidase activity in combination with specific drugs applied in clinical techniques of interventional pain management and local anaesthesia. Pain Physician 2008; 11:877-883.

25. Moore DC. The use of hyaluronidase in local and nerve block analgesia other than spinal block: 1520 cases. Anesthesiology 1951; 12:611-626.

26. McCleane GJ. Caudal epidural hyaluronidase can significantly reduce the pain associated with failed back surgery syndrome. Pain Clinic 2001; 13:119-123.

27. Geurts JW, Kallewaard JW, Richardson J, Groen GJ. Targeted methylprednisolone acetate/hyaluronidase/clonidine injection after diagnostic epiduroscopy for chronic sciatica: A prospective, 1-year follow-up study. Reg Anesth Pain Med 2002; 27:343-352.

28. Yousef AA, AS EL-D, Al-Deeb AE. The role of adding hyaluronidase to fluoroscopically guided caudal steroid and hypertonic saline injection in patients with failed back surgery syndrome: A prospective, double-blinded, randomized study. Pain Pract 2010; 10:548-553.

29. Choi EJ, Yoo YJ, Lee PB, Kim Y-C, Lee SC, Moon JY. A retrospective study to evalu- ate the effect of concentration of hypertonic saline on efficacy and safety of epidural adhesiolysis. Anesth Analg 2017; 124:2021-2029.

30. Kim SB, Lee KW, Lee JH, Kim MA, Kim $\mathrm{BH}$. The additional effect of hyaluronidase in lumbar interlaminar epidural injection. Ann Rehabil Med 2011; 35:405-411.

31. Rahimzadeh P, Sharma V, Imani F, et al. Adjuvant hyaluronidase to epidural steroid improves the quality of analgesia in failed back surgery syndrome: A prospective randomized clinical trial [published online ahead of print $\{A U$ : add date month $x x$, year $\}$. Pain Physician 2014; 17:E75-E82. \{AU: add doi\}.

32. Rapcan R, Kocan L, Mláka J, et al. A randomized, multicenter, double-blind, parallel pilot study assessing the effect of mechanical adhesiolysis vs adhesiolysis with corticosteroid and hyaluronidase administration into the epidural space during epiduroscopy. Pain Med 2018; 19:1436-1444.

33. Jamison DE, Hus E, Cohen SP. Epidural adhesiolysis: An evidence-based review. J Neurosurg Sci 2014; 58:65-76.

34. Gerdesmeyer L, Wagenpfeil S, Birkenmaier $C$, et al. Percutaneous epidural lysis of adhesions in chronic lumbar radicular pain: A randomized, doubleblind, placebo-controlled trial. Pain Physician 2013; 16:185-196.

35. Lee HK, Choi EJ, Lee PB, Nahm FS. Anaphylactic shock caused by the epidurally-administered hyalurinidase. Korean J Pain 2011; 24:221.

36. Kim TW, Lee JH, Yoon KB, Yoon DM. Allergic reactions to hyaluronidase in pain management - A report of three cases. Korean J Anesthesiol 2011; 60:57.

37. Kim J-H, Choi G-S, Ye Y-M, Nahm D-H, Park H-S. Acute urticaria caused by the injection of goat-derived hyaluronidase. Allergy Asthma Immunol Res 2009; 1:48-50.

38. Song YK, Kim YD, Kim JH. Acute allergic reaction caused by hyaluronidase used in the pain management: $A$ case report and literature review: a case report. Anesth Pain Med 2014; 9:174-178.

39. Dunn AL, Heavner JE, Racz G, Day M. Hyaluronidase: A review of approved formulations, indications and off-label use in chronic pain management. Expert Opin Biol Ther 2010; 10:127-131.

40. Fronza M, Muhr C, Calheiros da Silveira DS, et al. Hyaluronidase decreases neutrophils infiltration to the inflammatory site [published online ahead of print March 4, 2016]. Inflamm Res 2016. doi: 10.1007/sooo11-016-0935-0.

41. Huang Z, Zhao C, Chen Y, et al. Recombinant human hyaluronidase $\mathrm{PH}_{2} \mathrm{O}$ does not stimulate an acute inflammatory response and inhibits lipopolysaccharide-induced neutrophil recruitment in the air pouch model of inflammation. J Immuno 2014; 192:5285-5295.

42. Rosengren S, Dychter SS, Printz MA, et al. Clinical immunogenicity of $\mathrm{rH}$ $\mathrm{uPH}_{2} \mathrm{O}$, a hyaluronidase enabling subcutaneous drug administration. AAPS ] 2015; 17:1144-1156.

43. Birkenmaier C, Redeker J, Sievers B, Melcher C, Jansson V, Mayer-Wagner S. An evaluation of medications commonly used for epidural neurolysis procedures in a human fibroblast cell culture model. Reg Anesth Pain Med 2011; 36:140-144.

44. Schulze C, Bittorf T, Walzel H, Kundt G, Bader R, Mittelmeier W. Experimental evaluation of hyaluronidase activity in combination with specific drugs applied in clinical techniques of interventional pain management and local anaesthesia. Pain Physician 2008; 11:877-883.

45. Gourie-Devi M, Satish P. Hyaluronidase as an adjuvant in the treatment of cranial arachnoiditis (hydrocephalus and optochiasmatic arachnoiditis) complicating tuberculous meningitis. Acta Neurol Scand 1980; 62:368-381.

46. Gourie-Devi M, Satish P. Intrathecal hyaluronidase treatment of chronic spinal arachnoiditis of noninfective etiology. Surg Neurol 1984; 22:231-234.

47. Gourie-Devi M, Satishchandra P. Hyaluronidase as an adjuvant in the management of tuberculous spinal arachnoiditis. J Neurol Sci 1991; 102:105-111.

48. Knott LW, Katz J, Rubinstein LJ. Separate and combined effects of phenol, hyaluronidase, and dimethyl sulfoxide on the sciatic nerve of the rat: II. Chronic studies. Neurology 1969; 19:946-953.

49. Fabris LK, Šuput A, Gusic N, Mamontov P. Epidural adhesiolysis in the management of chronic low back pain in failed back surgery syndrome and in lumbar radicular pain: First year of experience at Pula General Hospital, Pula, Croatia -A Randomized Trial. Acta Med Croatica 2019; 73:57-64.

50. Heavner JE, Racz GB, Raj P. Percutaneous epidural neuroplasty: Prospective evaluation of $0.9 \% \mathrm{NaCl}$ versus $10 \%$ $\mathrm{NaCl}$ with or without hyaluronidase. Reg Anesth Pain Med 1999; 24:202-207. 
